\title{
Three-dimensional flow characteristics in ventricular assist devices: Impact of valve design and operating conditions
}

\author{
Christoph Benk, MSc, ${ }^{\mathrm{a}}$ Ramona Lorenz, MSc, ${ }^{\mathrm{b}}$ Friedhelm Beyersdorf, MD, PhD, ${ }^{\mathrm{a}}$ Jelena Bock, MSc, ${ }^{\mathrm{b}}$ \\ Rolf Klemm, MSc, ${ }^{a}$ Jan G. Korvink, PhD, ${ }^{\mathrm{c}, \mathrm{d}}$ and Michael Markl, PhD ${ }^{\mathrm{b}}$
}

\begin{abstract}
Objective: The use of paracorporeal ventricular assist devices has become a well-established procedure for patients with cardiogenic shock. However, implantation of ventricular assist devices is often associated with severe complications, such as thrombosis inside the ventricular assist device and subsequent embolic events. It was the purpose of this study to use flow-sensitive 4-dimensional magnetic resonance imaging for a detailed analysis of the 3-dimensional (3D) flow dynamics inside a clinical routine ventricular assist device and to study the effect of different system adjustments and a new valve design on flow patterns.
\end{abstract}

\begin{abstract}
Methods: A routinely used clinical paracorporeal ventricular assist device was integrated into a magnetic resonance-compatible mock loop. Flow-sensitive 3D magnetic resonance imaging was performed to measure time-resolved 3-directional flow velocities (spatial resolution $\sim 1.2 \mathrm{~mm}$, temporal resolution $=42.4 \mathrm{~ms}$ ) in the entire device under ideal conditions (full fill, full empty, ejection fraction $=88 \%$ ), insufficient filling (ejection fraction $=81 \%$ ), and insufficient emptying (ejection fraction $=67 \%$ ) of the pump chamber. In addition, a new valve design was evaluated. Flexible control and monitoring of pressures at inlet and outlet were used to generate realistic boundary conditions.
\end{abstract}

Results: Flow pattern changes for different operating conditions were clearly identified and included reduced velocities during systolic outflow for impaired filling (78\% reduction in pump flow compared with optimal operating conditions) and impaired clearing of the pump chamber for insufficient emptying (52\% reduction). For all operating conditions, 3D visualization revealed vortex flow inside the ventricular assist device at typical locations of thrombus formation near the valve systems. Most noticeably, the new valve design provided similar global ventricular assist device function (pump flow $3.6 \mathrm{~L} / \mathrm{min}$ ), but vortex formation was eliminated.

Conclusions: The results of this study provide insight into the mechanisms underlying possible thrombus formation inside a ventricular assist device and the effect of different system adjustments. The presented methods may permit the optimization of future ventricular assist device systems with respect to optimal flow conditions. (J Thorac Cardiovasc Surg 2011;142:1019-26)

Video clip is available online.

Acute heart failure requires immediate therapeutic intervention, including the implantation of total artificial hearts or ventricular assist devices (VADs) replacing or assisting

\footnotetext{
From the Department of Cardiovascular Surgery, ${ }^{\text {a }}$ University Hospital, Freiburg, Germany; Department of Radiology, ${ }^{\mathrm{b}}$ Medical Physics, University Hospital, Freiburg, Germany; Institute of Microsystems Engineering, ${ }^{\mathrm{c}}$ Laboratory for Simulation, University Freiburg, Freiburg, Germany; and Freiburg Institute for Advanced Studies, ${ }^{\mathrm{d}}$ University Freiburg, Freiburg, Germany.

Funding: Deutsche Forschungsgemeinschaft Grant MA 2383/5-1 and Bundesministerium für Bildung und Forschung Grant 01EV0706.

Disclosures: Authors have nothing to disclose with regard to commercial support.

Received for publication Oct 5, 2010; revisions received Jan 10, 2011; accepted for publication Jan 29, 2011; available ahead of print March 14, 2011.

Address for reprints: Friedhelm Beyersdorf, MD, PhD, University Hospital Freiburg, Department of Cardiovascular Surgery, Hugstetter Strasse 55, D-79106 Freiburg (E-mail: friedhelm.beyersdorf@uniklinik-freiburg.de).

$0022-5223 / \$ 36.00$

Copyright (c) 2011 by The American Association for Thoracic Surgery doi:10.1016/j.jtcvs.2011.01.058
}

the failing heart. In recent years, a number of different systems have been introduced and have substantially improved the outcome and survival in patients with heart failure. ${ }^{1-3}$ In addition to devices that are fully implanted into the chest, paracorporeal ventricular assist devices (PVADs) are also an established treatment option for uni- or biventricular support in acute heart failure, especially in children. ${ }^{4,5}$ PVAD implantation is often performed on a temporary basis to serve as a bridge to transplantation or bridge to recovery. ${ }^{6-11}$

The role of the VAD in the management of heart failure is expanding, but there is still a high risk for complications. The most common described complications are bleeding, infection, pericardial tamponade, respiratory events, technical problems, and neurologic events. ${ }^{2,3}$ Thrombosis in the valve region of a pulsatile VAD may cause embolic neurologic events with a high rate of morbidity and mortality. Most often, fibrin or thrombus formation in the VAD is generated near the inflow or outflow valve or in the pump chamber as the result of insufficient anticoagulation or a poorly adjusted system. ${ }^{12,13}$ In addition, particular flow characteristics inside 


$$
\begin{aligned}
& \text { Abbreviations and Acronyms } \\
& \begin{aligned}
\mathrm{EF} & =\text { ejection fraction } \\
\mathrm{MR} & =\text { magnetic resonance } \\
\mathrm{MRI} & =\text { magnetic resonance imaging } \\
\mathrm{PIV} & =\text { particle imaging velocimetry } \\
\mathrm{PVAD} & =\text { paracorporeal ventricular assist device } \\
\text { 3D } & =3 \text {-dimensional } \\
\text { 4D } & =4 \text {-dimensional } \\
\mathrm{VAD} & =\text { ventricular assist device }
\end{aligned}
\end{aligned}
$$

the VAD, such as vortex formation, flow reversal, and thus increased residence times, may explain the high rate of thromboembolic events after VAD implantation and the described changes in von Willebrand factor. ${ }^{13}$

A number of studies based on different imaging modalities (ultrasound, particle imaging velocimetry [PIV]) or numeric simulations (computational fluid dynamics) have investigated flow patterns inside assist devices. ${ }^{14,15}$ Recently, the application of flow-sensitive 4-dimensional (4D) magnetic resonance imaging (MRI) ${ }^{16}$ was used for the ex vivo assessment of pulsatile 3-dimensional (3D) flow characteristics in a routine VAD implantation. ${ }^{17}$

However, to date no systematic evaluation of different VAD designs (eg, valve systems at inlet and outlet) on flow characteristics, and thus VAD performance, has been reported. In addition, the effect of different operating conditions, often observed in the clinical routine (optimal vs suboptimal driving of the device), on regional flow characteristics, and thus risk for thrombus formation, is poorly understood. ${ }^{12,13,18}$

The purpose of this study was to use flow-sensitive 4D MRI for a detailed analysis of 3D flow dynamics and function of the inlet and outlet valve systems in a clinical routine VAD for different system adjustments representing optimal and impaired operating conditions. In a second set of experiments, the effect of different valve designs on regional flow patterns and valve dynamics were directly compared. Compared with previous studies, an improved magnetic resonance (MR)-compatible flow circuit was developed, including blood mimicking fluid and flexible pressure control and monitoring to provide physiologic pre- and afterload conditions.

\section{MATERIALS AND METHODS \\ Magnetic Resonance-Compatible Ventricular Assist Device Flow Circuit}

The experimental setup is schematically illustrated in Figure 1. A clinically used VAD (MEDOS VAD-Pump-Chamber, MEDOS Medizintechnik AG, Stolberg, Germany; chamber size $=60 \mathrm{~mL})^{4,5,19,20}$ was integrated into an MR-compatible flow circuit that could be placed directly inside a routine 3 Tesla MR system (TRIO, Siemens, Germany). Pulsatile VAD flow was generated using pulsatile pneumatic pressure created by a routine VAD control unit (MEDOS VAD-Driving Unit, MEDOS Medi-

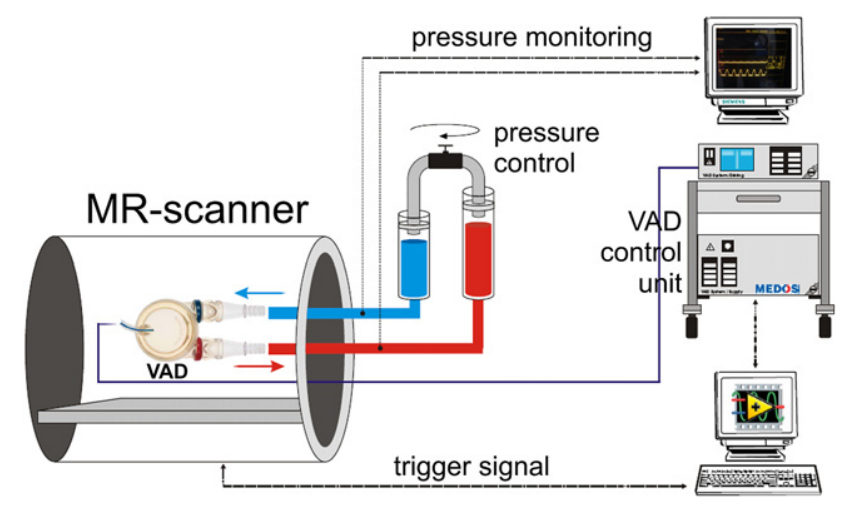

FIGURE 1. Schematic representation of measurement setup for in vitro 3D flow analysis via MRI in the VAD. The VAD control unit and pressure monitoring were placed outside the MR scanner room and connected to the VAD flow circuit inside the scanner by long (8-m) tubing. The mock loop used for the adjustment of pre- and afterload conditions was placed on the patient table next to the VAD. $M R$, Magnetic resonance; $V A D$, ventricular assist device.

zintechnik AG). ${ }^{17}$ As an improvement to our previous setup, a mock loop was integrated to permit flexible pressure control via adjustment of different water levels at inlet and outlet in combination with an adjustable valve to simulate nearly physiologic pre- and afterload conditions similar to strategies reported by Timms and colleagues. ${ }^{21}$ A patient monitor (Sirecust, Siemens, Erlangen, Germany) was used to observe the pressure conditions at the inlet and outlet of the VAD. Blood mimicking fluid was used as blood substitute, a mixture of $60 \%$ distilled water and $40 \%$ glycerol closely reproducing the kinematic viscosity of blood $\left(\nu=0.003 \mathrm{~m}^{2} / \mathrm{s}\right) .{ }^{22}$

\section{Magnetic Resonance 4D Flow Measurements}

Time-resolved 3D phase contrast MRI with 3-directional velocity encoding (flow-sensitive 4D MRI) was used to measure the temporal evolution of complex 3-directional flow in a 3D volume. ${ }^{16,23}$ The fully non-magnetic and non-metallic model systems and synchronization of the MR data acquisition with the cyclic device function were used for the systematic investigation of fluid dynamics inside a VAD as described previously. ${ }^{17}$

All MR experiments were performed using a 3T MRI system (Trio, Siemens). Flow-sensitive 4D data were acquired in a coronal 3D volume encompassing the entire VAD (field of view $=154 \times 280 \mathrm{~mm}$, slab thickness $=62.4 \mathrm{~mm}$ ) with a spatial resolution of $1.1 \times 1.2 \times 1.3 \mathrm{~mm}^{3}$ and temporal resolution of $42.4 \mathrm{~ms}{ }^{16,17,24}$ Further imaging parameters were as follows: echo time $=2.7 \mathrm{~ms}$, repetition time $=5.4 \mathrm{~ms}$, velocity sensitivity $=200 \mathrm{~cm} / \mathrm{s}$, flip angle $=15$ degrees, and total scan time $=28$ minutes, 6 seconds.

\section{Operating Conditions}

To investigate the effect of different operating conditions, flow-sensitive 4D MRI was performed under ideal conditions (complete filling and emptying), insufficient filling (expansion), and insufficient emptying (contraction) of the pump chamber. The different VAD operating conditions were achieved by adjusting the systolic (Pressure Systole $_{\text {) and diastolic }}$

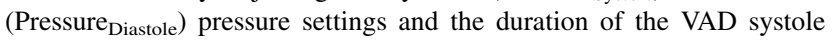
(Time $_{\text {Systole }}$ ) at the VAD control while visually inspecting the performance of the VAD pump chamber for optimal and impaired VAD pump function. The corresponding pump unit settings and achieved preload and afterload pressures for all conducted flow experiments are summarized in Table 1. The ejection fraction (EF) for all operation conditions was calculated as $\mathrm{EF}=\left(\mathrm{A}_{\text {Diastole }}-\mathrm{A}_{\text {Systole }}\right) / \mathrm{A}_{\text {Diastole }}$ by segmenting the VAD chamber area 
TABLE 1. Settings of the ventricular assist device control unit for the different operating conditions, including the preload and afterload conditions for the different ventricular assist device flow experiments

\begin{tabular}{|c|c|c|c|c|}
\hline VAD settings & Optimal conditions & Insufficient filling & Insufficient emptying & $\begin{array}{c}\text { Optimal conditions } \\
\text { and new valve design }\end{array}$ \\
\hline Pressure $_{\text {Systole }}$ & $230 \mathrm{~mm} \mathrm{Hg}$ & $230 \mathrm{~mm} \mathrm{Hg}$ & $190 \mathrm{~mm} \mathrm{Hg}$ & $230 \mathrm{~mm} \mathrm{Hg}$ \\
\hline Pressure $_{\text {Diastole }}$ & $-40 \mathrm{~mm} \mathrm{Hg}$ & $-10 \mathrm{~mm} \mathrm{Hg}$ & $-40 \mathrm{~mm} \mathrm{Hg}$ & $-40 \mathrm{~mm} \mathrm{Hg}$ \\
\hline Time $_{\text {Systole }}$ & $35 \%$ & $50 \%$ & $26 \%$ & $35 \%$ \\
\hline Frequency & 60 & 60 & 60 & 60 \\
\hline Pump flow & $\sim 3.6 \mathrm{~L} / \mathrm{min}$ & $<3.0 \mathrm{~L} / \mathrm{min}$ & $\sim 3.0 \mathrm{~L} / \mathrm{min}$ & $\sim 3.6 \mathrm{~L} / \mathrm{min}$ \\
\hline Preload & 3-6 mm Hg & $3-5 \mathrm{~mm} \mathrm{Hg}$ & $5-6 \mathrm{~mm} \mathrm{Hg}$ & $1-5 \mathrm{~mm} \mathrm{Hg}$ \\
\hline $\begin{array}{r}\text { Afterload (systolic pressure, } \\
\text { diastolic pressure, MAP) }\end{array}$ & $135,-4,42 \mathrm{~mm} \mathrm{Hg}$ & $140,-4,43 \mathrm{~mm} \mathrm{Hg}$ & $135,-3,43 \mathrm{~mm} \mathrm{Hg}$ & $137,-7,41 \mathrm{~mm} \mathrm{Hg}$ \\
\hline
\end{tabular}

$V A D$, Ventricular assist device; $M A P$, mean arterial pressure.

during maximum compression $\left(\mathrm{A}_{\text {Systole }}\right)$ and expansion $\left(\mathrm{A}_{\text {Diastole }}\right)$, as shown in Figure 2.

For all VAD settings, additional measurements with the pump turned off (flow-off data), but otherwise identical data acquisition parameters, were performed.

\section{Ventricular Assist Device Valve Designs}

The standard VAD valve design consisted of 3 bulbs at the inlet and outlet side that were modeled according to the normal aortic root geometry, including a model of the sinuses of Valsalva. The 3 cusps were made of polyurethane. The inserted 3-leaflet valves were designed and adjusted in construction and function according to the natural aortic valve. In addition, a VAD with a new valve design was evaluated under ideal conditions, that is, complete filling and emptying. The new valve design also consisted of 3 valve cusps, which, however, were embedded in a differently shaped geom- etry with a symmetric cylindrical shape at inlet and outlet. The VAD pump chambers were identical for both designs. The 3 sinuses of Valsalva, used in the standard valve design, were therefore omitted in the new valve.

\section{Data Analysis}

Flow-off data were subtracted from the velocity images to remove unwanted background offsets. Correction for velocity aliasing and noise masking was performed as described previously. ${ }^{25}$

Three-dimensional visualization software (EnSight, Computational Engineering International, Inc, Apex, NC) was used to display 3D VAD geometry using iso-surface rendering of the magnitude data (Figure 2). The measured 3D flow characteristics inside the VAD were visualized using 3D particle traces resembling traces of massless virtual particles that were released and path integrated in the measured time-resolved velocity vector field. A simultaneous visualization of spatial and dynamic changes

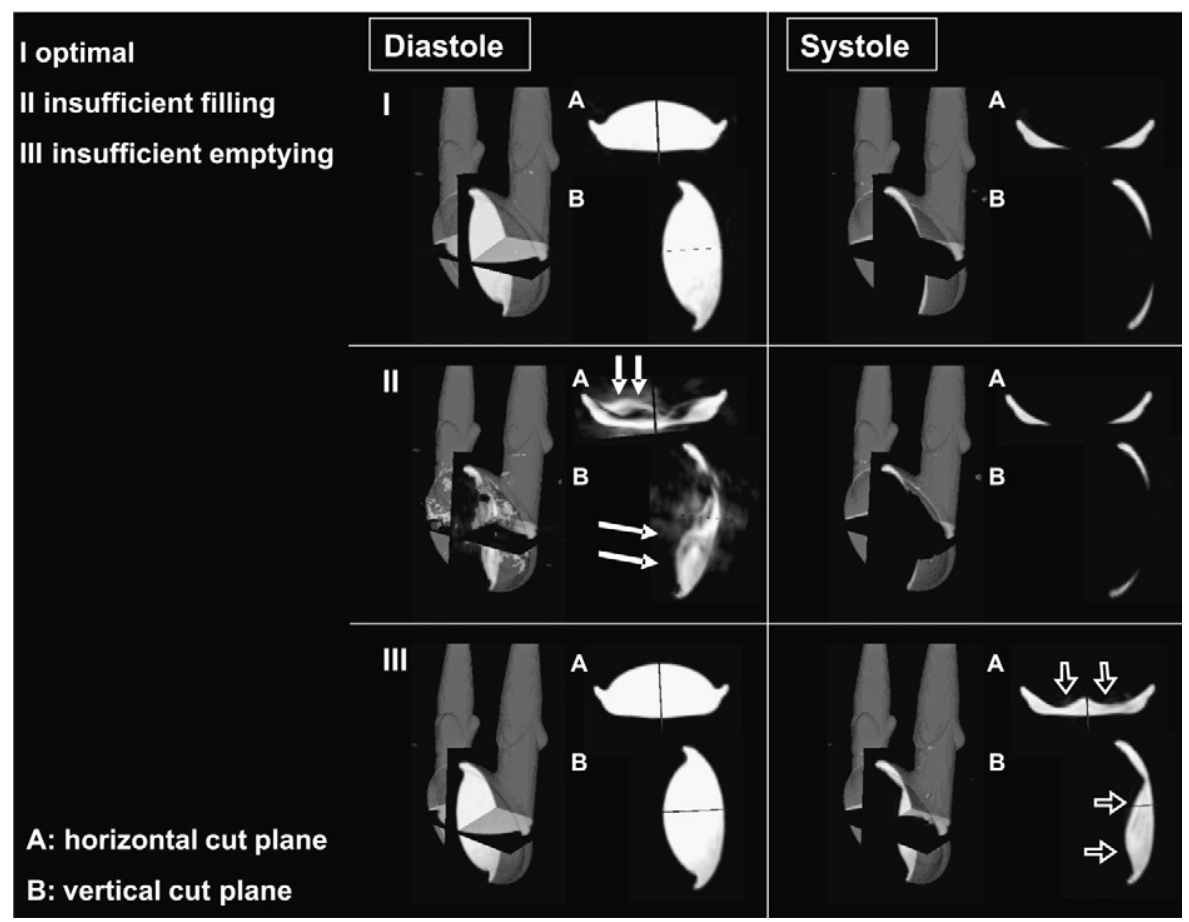

FIGURE 2. VAD geometry and pump chamber volume during maximum filling (diastole) and contraction (systole) for different operating conditions. The gray shaded surface represents the 3D VAD geometry. For the analysis of pump chamber volume for different operating conditions, analysis planes transecting the VAD chamber in horizontal (A) and vertical (B) orientation were used. The maximal diastolic and minimal systolic VAD pump chamber volumes were quantified and used to calculate the VAD EF. 
in flow patterns and VAD geometry was achieved by superimposing 3D particle traces and 3D iso-surface (Figures 3 and 4). ${ }^{15,16}$

To further evaluate the effect of operation conditions and valve design, the velocity profiles of flow through the valves were analyzed as colorcoded velocity maps (Figure 5). In addition, flow quantification was performed in 2-dimensional analysis planes that were manually positioned distal to the inlet and outlet valves of the VAD.

\section{RESULTS}

Three-dimensional VAD geometry and flow characteristics were successfully measured in all experiments. On the basis of the combination of the mock loop and real-time pressure monitoring, consistent pressure conditions (systolic afterload $=135-140 \mathrm{~mm} \mathrm{Hg}, 1-6 \mathrm{~mm} \mathrm{Hg}$ preload) at the inlet and outlet could be achieved as summarized in Table 1 .

\section{Ventricular Assist Device Geometry}

Figure 2 illustrates the 3D VAD geometry during maximum contraction (systole) and expansion (diastole) of the pump chamber for all evaluated operating conditions. Systolic and diastolic chamber shapes are shown in 2 orthogonal planes transecting the VAD in vertical (Figure 2, B) and horizontal orientation (Figure 2, A). For insufficient filling, diastolic chamber expansion was clearly reduced (Figure 2, middle row, solid white arrows, $\mathrm{EF}=81 \%$ ) compared with optimal operating conditions (Figure 2, top row, $\mathrm{EF}=88 \%$ ). For impaired emptying, an asymmetric and incomplete contraction of the chamber volume during systole is clearly evident (Figure 2, lower row, open white arrows, $\mathrm{EF}=67 \%$ )

\section{Ventricular Assist Device Flow Characteristics}

Three-dimensional flow visualization was successfully performed for all VAD measurements as illustrated in Figure 3, which depicts VAD flow characteristics during a complete pump cycle for the new valve design under optimal operating conditions. Characteristic flow patterns and
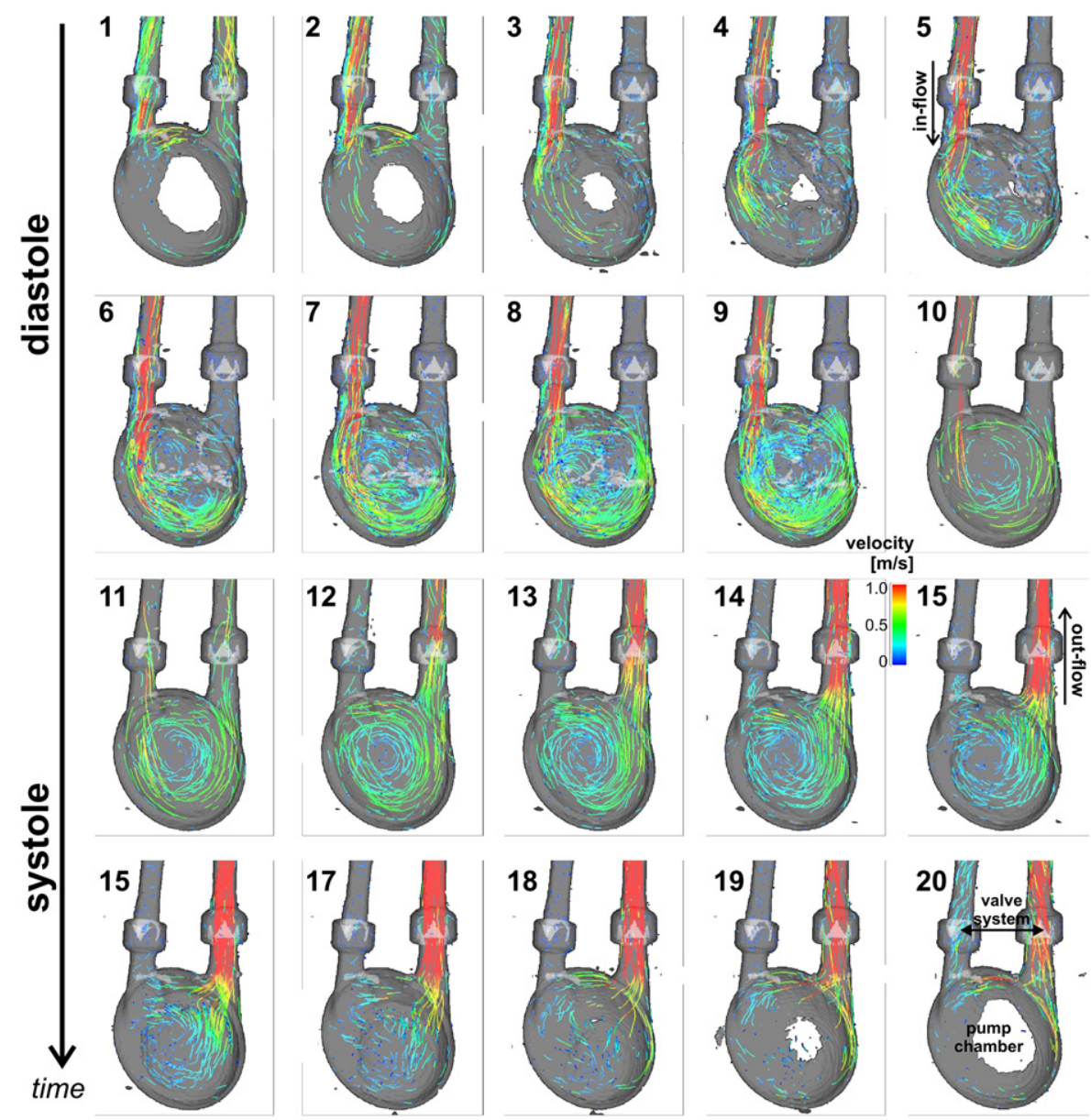

FIGURE 3. Three-dimensional flow visualization using time-resolved 3D particle traces in the VAD during a complete pump cycle. The series of images represent the development of flow patterns during 1 VAD cycle for the VAD with the new valve design under optimal operating conditions. The time frame in the VAD cycle is indicated by the numbers in the top left corner of each image, and flow dynamics are depicted with a temporal resolution (time between 2 successive images) of $42.4 \mathrm{~ms}$. The gray shaded iso-surface rendering represents the VAD geometry including pump chamber and valve systems at inlet and outlet. Color coding $=$ local absolute flow velocities. 

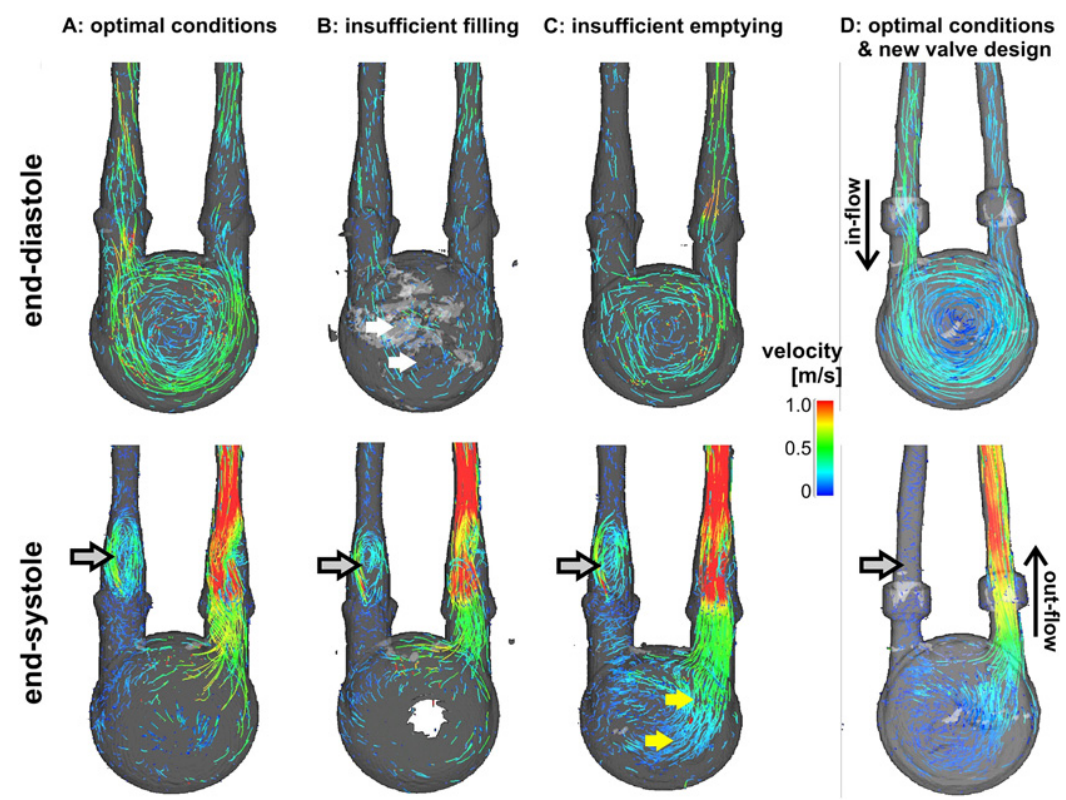

FIGURE 4. Three-dimensional flow characteristics inside the VAD during maximum chamber expansion (end diastole) and peak outflow phase (end systole). The gray shaded area represents an iso-surface rendering of the magnitude data and shows the VAD geometry including pump chamber and valve systems at inlet and outlet. The effect of different operating conditions in the same VAD clearly resulted in considerable differences in VAD function (A-C). Vortex formation proximal to the inlet valve was clearly visible for all operating conditions but absent for the new valve design (D, gray arrows). The comprehensive flow dynamics during the entire VAD cycle are clearly shown in Video 1. Color coding = local absolute flow velocities.

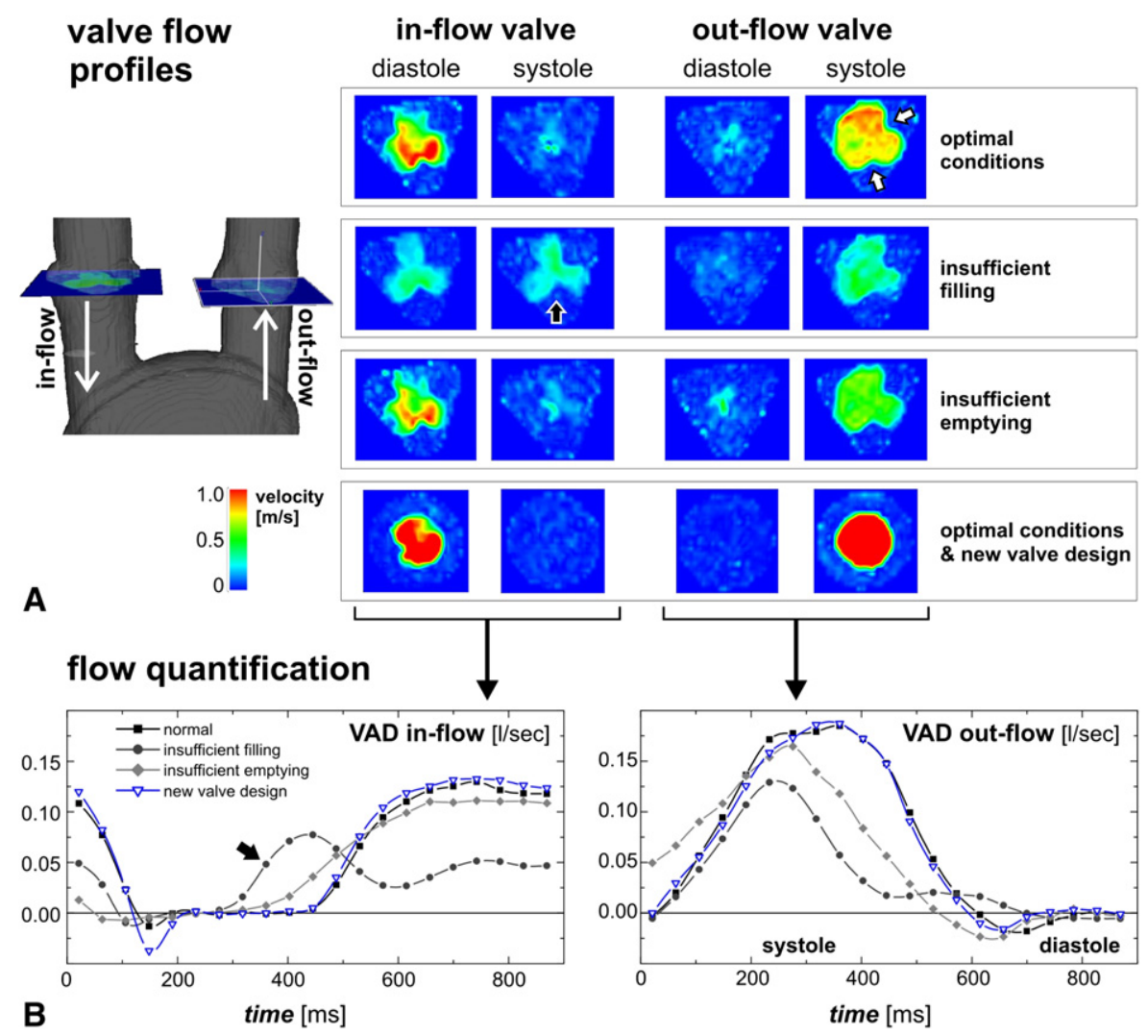

FIGURE 5. A, Fluid velocity distribution in analysis planes at the level of the VAD inflow and outflow valves. The individual images show the velocity profiles during peak inflow (diastole) and outflow (systole). B, Quantified time-resolved flow during 1 VAD pump cycle distal to the valve at the inlet (left) and outlet (right). Color coding $=$ local absolute flow velocities. VAD, Ventricular assist device. 
the temporal evolution of flow are depicted by 3D particle traces and illustrate diastolic inflow (Figure 3, top row), complex swirling and circular flow patterns during pump chamber filling (Figure 3, second row), chamber emptying during systole (Figure 3, third row), and outflow with high velocities during late systole (Figure 3, lower row). Visualization quality was similar for all other investigated operation conditions and VADs.

\section{Operating Conditions and Effect of Valve Design}

As summarized in Figure 4, flow pattern changes for different operating conditions were clearly evident. Note the different geometries of the standard valve housing (aortic root shape) and the new design (cylindrical shape). Compared with normal operating conditions, insufficient VAD filling resulted in reduced velocities during systolic outflow. The low numbers of end-diastolic particle traces inside the pump chamber (Figure 4, white arrows) indicate reduced inflow compared with optimal conditions.

Insufficient emptying led to impaired clearing of the pump chamber (Figure 4, yellow arrows) while maintaining high systolic outflow velocities. Potentially thrombogenic vortex formation proximal to the inlet valve was clearly visible for all operating conditions (Figure 4, gray arrows).

A novel valve design using a differently shaped valve support region resulted in improved VAD performance. In addition to improved filling of the outflow cannula, vortex formation proximal to the inflow valve was virtually eliminated.

\section{Valve Flow Profiles and Quantification}

The results of the flow profile analysis and quantification at the level of the inlet and outlet valves are summarized in Figure 5.

The standard VAD valves open in a star-shape at both inlet and outlet as determined by the valve geometry. Despite normal operating conditions (Figure 5, A, top row), the valve opening area and velocity distribution appeared asymmetric, and only 1 of the valvular cusps was fully opened at the outflow valve (Figure 5, white arrows). Impaired operating conditions (Figure 5, $A$, rows 2 and 3 ) clearly altered the shape and size of the valve opening area at inlet and outlet. The worst performance was observed for insufficient filling demonstrating reduced flow velocities during the entire pump cycle and altered valve dynamics, including opened inlet valves during systole (Figure 5, black arrows). The new valve design (Figure 5, $A$, row 4 ) resulted in a more symmetric valve opening area and homogenous velocity distributions at inlet and outlet.

As expected, flow quantification revealed impaired performance for insufficient emptying (Figure 5, $B$, diamonds) and particularly insufficient filling (Figure 5, circles). Normal operating conditions for the standard VAD resulted in a total VAD outflow of $3.7 \mathrm{~L} / \mathrm{min}$. Insufficient filling and emptying clearly reduced VAD function by $78 \%$ and $52 \%$ to $1.9 \mathrm{~L} / \mathrm{min}$ and $2.8 \mathrm{~L} / \mathrm{min}$, respectively. The VADs with standard (squares) and novel (open triangles) valve design demonstrated almost identical flow behavior (total flow $=3.6 \mathrm{~L} / \mathrm{min}$ ).

\section{DISCUSSION}

Flow-sensitive MRI and advanced 3D flow visualization methods were used to derive a comprehensive picture of flow dynamics within the VAD system for different operating conditions and valve designs. The data could successfully be used to evaluate the dynamics of pump chamber volume and valve function at inlet and outlet. The identification of complex flow patterns, such as vortex formation, and the observed differences in valve opening and closing dynamics point toward the potential of the presented methodology to further deepen the understanding of VAD function.

As expected, different operating conditions for the same VAD resulted in considerable altered VAD function. The results clearly showed the importance of an optimally adjusted system to avoid filling and emptying problems of the artificial ventricle that prevent achieving a perfect washout of the system. In addition to the expected reduction of total VAD pump performance for insufficient filling or emptying, that is, poor washout of the ventricle, marked alterations in valve function (Figure 5, A), and pulsatile flow dynamics (Figure $5, B$ ) were observed.

The highest incidence of inadequate system performance in clinical routine results from insufficient filling of the artificial ventricle. These findings therefore indicate that such operating conditions not only result in an inadequate support of the patient but also affect valve function and regional flow patterns. We hypothesize that the observed low velocities near the valve systems and prolonged valve opening duration may result in increased risk of fibrin and thrombus accumulation.

For the standard VAD, complex flow and particularly pronounced end-systolic vortex formation were seen independently of system adjustments. These findings provide practical insight regarding a potential mechanism for thrombus formation inside a VAD. Vortex flow can result in altered blood shear rates and increased residence times, which are known to promote the development of thrombi. Although no direct link between the flow patterns and the existence of thrombi was demonstrated, the location of the flow vortex near the inlet valve systems corresponds well with the region of frequent thrombus occurrence observed in clinical routine.

Initial results from flow pattern analysis in a VAD with an alternative valve design indicated an improved washout and, most noticeably, the complete absence of vortex flow near the valve systems. In addition, the altered valve geometry resulted in a more symmetric circular valve opening area. We 
hypothesize that these geometric changes resulted in the more homogenous velocity distribution inside the valve and consequently less flow disturbance and reduced risk for vortex formation. These preliminary findings indicate the potential of in vitro experiments to optimize VAD design.

It should be noted that the new valves are still an experimental design and have not yet been used clinically. However, our results show improved flow characteristics for this new type of valve. The major limitation of the standard valve design has been thrombus formation. ${ }^{26}$ In our own clinical experience, thrombi were especially found in the sinuses of Valsalva. This thrombin generation was the main reason for pump exchange in up to $50 \%$ of patients. However, thrombus formation is also a major complication in other paracorporeal pulsatile VADs. ${ }^{27}$

These findings regarding regional flow alterations should be interpreted with care. Areas of turbulence or complex flow as seen near the VAD valves may lead to pockets of stasis and increased thrombogenicity. However, areas of complex flow and thus potentially increased shear stress may also lead to an acquired factor VIII deficiency and therefore less thrombogenicity and increased bleeding tendency. Elevated shear stress may also result in increased hemolysis without increased thrombogenicity. Moreover, Virchow's triad of thrombus formation involves an injury to the vessel, a change in the blood's innate thrombogenicity, and an alteration in the flow pattern. It is important to note that the presented model only addresses 1 of these 3 components and therefore has limited ability to predict the actual clinical outcome. Animal studies or experiments with real blood are therefore needed to evaluate the true impact of the observed flow alterations.

The presented technique implies a number of limitations. All measurements were performed using an in vitro mock loop model, filled with a fluid similar to blood, with flexibly adjustable pressure conditions. Although care was taken to simulate in vivo conditions as closely as possible, the pressure conditions at inlet and outlet and the total pump flow were somewhat suboptimal. Therefore, the interpretation of the results with respect to the situation in the clinical routine must be considered carefully. The in vitro model allowed a maximum pump frequency of $60 / \mathrm{min}(\sim 3.6$ $\mathrm{L} / \mathrm{min}$ ) because of the capacitance of the long pneumatic tubings that were necessary to connect the VAD to the pump unit outside the scanner room. The range of possible heart rates and pressure conditions was thus limited. Moreover, the use of real blood instead of blood mimicking fluid may alter the observed flow patterns in the VAD. Future studies are therefore needed with an improved model system that permits higher VAD output and frequencies to systematically evaluate the influence of these parameters on VAD function. In addition, the model could be refined to permit evaluation of the influence of individual VAD cannula positions relative to the heart.
A further limitation of the presented in vitro setup is related to the isolated evaluation of the VAD under different operating conditions or with a novel valve design. In investigations of optimal pumping specifications, the influence of left ventricular ejection on filling and washing of the pump has been ignored. Future experiments should include animal studies that could provide an ideal setting to model the complete system (left ventricle plus VAD) and its reaction to different operating conditions and VAD designs. ${ }^{28}$

The in vitro experiments presented in this study were based on a pulsatile VAD that was introduced several years ago. Given the rapid advancements in VAD technology, specifically the current transition from pulsatile to continuous flow systems, future studies should include different and continuous-flow devices. ${ }^{29,30}$ A drawback of the MRbased device characterization is related to the need for non-magnetic and largely non-metallic assist devices. The presence of magnetic or metallic materials could make the measurements impossible or result in substantial imaging artifacts. As a remedy, existing devices might be replicated using MR-compatible materials and then used for in vitro flow assessment.

Because no imaging technology exists to measure 3D flow inside a clinical VAD implanted in a patient, the in vitro setup may provided a useful model system using real VAD equipment and near physiologic conditions. Alternatively, numeric simulations using computational fluid dynamics have been applied in a number of studies. ${ }^{14,15}$ However, boundary conditions are often simplified, and it is difficult to accurately model the clinical equipment. The advantage of the presented approach is related to the use of a standard clinical VAD and pump units and real measurement of time-resolved 3-directional fluid velocities.

It should be noted that the method used for 3D visualization of fluid flow (time-resolved particle traces) did not involve true physical particles that were present in the fluid in the VAD. Instead, a computer algorithm was used that traced the temporal behavior of virtual massless particles that were released inside the VAD. By repeated virtual particle emission over time, the calculated traces represent a collection of continuously propagating streaks that travel through the flow field according to the measured speeds and directions of flow. The animation of these traces (Video E1) provides a visual impression of time-resolved fluid flow. ${ }^{26}$

An alternative modality to investigate 3D fluid dynamics in model systems is provided by optical imaging techniques, such as PIV. ${ }^{15}$ A number of studies have shown that PIV can provide highly accurate depiction of fluid dynamics by tracing the evolution of suspended particles in a dynamically changing fluid. However, disadvantages of PIV include the need for matching the refraction index of the model systems and the fluid under investigation. These limitations do not apply to flow-sensitive MRI, which can be used to measure flow velocities in nontransparent models 
and different types of fluid. Further, PIV imaging is timeconsuming (several hours of scan time) and requires extensive postprocessing to derive particle traces. MRI measurements of the pulsatile 3D flow field, on the other hand, can be performed on the order of 30 minutes and are thus advantageous if different operating conditions are successively evaluated in 1 imaging session. Disadvantages of MRI compared with PIV include the requirement of fully non-magnetic and non-metallic model systems and the high cost of operating an MR system.

\section{CONCLUSIONS}

We presented the first evaluation of flow dynamics of a VAD connected to a mock loop under different operating conditions. Our findings indicate the feasibility of in vitro VAD analysis to improve the understanding of flow characteristics, pump chamber geometry, and valve dynamics under different system adjustments. Direct comparison with a new valve design showed the potential of this technique to improve the VAD designs under in vitro conditions without the need for complex computer simulations or animal studies.

\section{References}

1. Baughman KL, Jarcho JA. Bridge to life-cardiac mechanical support. $N$ Engl J Med. 2007;357:846-9.

2. Kirklin JK, Naftel DC, Stevenson LW, Kormos RL, Pagani FD, Miller MA, et al. INTERMACS database for durable devices for circulatory support: first annual report. J Heart Lung Transplant. 2008;27:1065-72.

3. Westaby S, Siegenthaler M, Beyersdorf F, Massetti M, Pepper J, Khayat A, et al. Destination therapy with a rotary blood pump and novel power delivery. Eur J Cardiothorac Surg. 2010;37:350-6.

4. Schmid C, Debus V, Gogarten W, Löher A, Drees G, Scheld HH, et al. Pediatric assist with the Medos and Excor systems in small children. ASAIO J. 2006;52: 505-8.

5. Kaczmarek I, Mair H, Groetzner J, Sachweh J, Oberhoffer M, Fuchs A, et al. Mechanical circulatory support in infants and adults with the MEDOS/HIA assist device. Artif Organs. 2005;29:857-60.

6. Kirklin JK, Holman WL. Mechanical circulatory support therapy as a bridge to transplant or recovery (new advances). Curr Opin Cardiol. 2006;21:120-6.

7. Farrar DJ. The Thoratec ventricular assist device: a paracorporeal pump for treating acute and chronic heart failure. Semin Thorac Cardiovasc Surg. 2000;12: 243-50.

8. Jaroszewski DE, Marranca MC, Pierce CN, Wong RK, Steidley ED, Scott RL, et al. Successive circulatory support stages: a triple bridge to recovery from fulminant myocarditis. J Heart Lung Transplant. 2009;28:984-6.

9. Kirsch M, Vermes E, Damy T, Nakashima K, Sénéchal M, Boval B, et al. Singlecentre experience with the Thoratec paracorporeal ventricular assist device for patients with primary cardiac failure. Arch Cardiovasc Dis. 2009;102:509-18. Epub 2009 May 23.

10. Pages ON, Aubert S, Combes A, Luyt CE, Pavie A, Léger P, et al. Paracorporeal pulsatile biventricular assist device versus extracorporal membrane oxygenationextracorporal life support in adult fulminant myocarditis. J Thorac Cardiovasc Surg. 2009; 137:194-7.
11. Slaughter MS, Silver MA, Farrar DJ. A new method of monitoring recovery and weaning the Thoratec left ventricular assist device. Ann Thorac Surg. 2001;71: 215-8.

12. Decoene C, Fayad G, Al-Ruzzeh S, Modine T, Crepin F, Pol A, et al. Right ventricular assist device thrombosis during biventricular heart assistance. Perfusion. 2004;19:365-7.

13. Heilmann C, Geisen U, Benk C, Berchtold-Herz M, Trummer G, Schlensak C, et al. Haemolysis in patients with ventricular assist devices: major differences between systems. Eur J Cardiothorac Surg. 2009;36:580-4.

14. Finocchiaro T, Heinke S, Behbahani M, Lessmann M, Laumen M, Steinseifer U, et al. Methods of design, simulation, and control for the development of new VAD/TAH concepts. Biomed Tech (Berl). 2009;54:269-81. Review.

15. Sato K, Orihashi K, Kurosaki T, Tokumine A, Fukunaga S, Ninomiya S, et al. Analysis of flow patterns in a ventricular assist device: a comparative study of particle image velocimetry and computational fluid dynamics. Artif Organs. 2009;33:352-9.

16. Markl M, Harloff A, Bley TA, Zaitsev M, Jung B, Weigang E, et al. Timeresolved 3D MR velocity mapping at 3T: improved navigator-gated assessment of vascular anatomy and blood flow. J Magn Reson Imaging. 2007;25:824-31.

17. Markl M, Benk C, Klausmann D, Stalder AF, Frydrychowicz A, Hennig J, et al. Three-dimensional magnetic resonance flow analysis in a ventricular assist device. J Thorac Cardiovasc Surg. 2007;134:1471-6.

18. Goldstein DJ. Coagulation and thrombotic complications associated with ventricular assist devices. Clin Adv Hematol Oncol. 2005;3:766-8.

19. Thuaudet S. The Medos ventricular assist device system. Perfusion. 2000;15: 337-43.

20. Reul H. The MEDOS/HIA system: development, results, perspectives. Thorac Cardiovasc Surg. 1999;47(Suppl. 2):311-5.

21. Timms D, Hayne M, McNeil K, Galbraith A. A complete mock circulation loop for the evaluation of left, right, and biventricular assist devices. Artif Organs. 2005;29:564-72.

22. Holton AD, Walsh EG, Brott BC, Venugopalan R, Hershey B, Ito Y, et al. Evaluation of in stent stenosis by magnetic resonance phase-velocity mapping in nickel-titanium stents. J Magn Reson Imaging. 2005;22:248-57.

23. Whitehead KK, Sundareswaran KS, Parks WJ, Harris MA, Yoganathan AP, Fogel MA. Blood flow distribution in a large series of patients having the Fontan operation: a cardiac magnetic resonance velocity mapping study. $J$ Thorac Cardiovasc Surg. 2009;138:96-102.

24. Lorenz R, Bock J, Stalder AF, Markl M. Novel closed circuit MR compatible pulsatile pump system using a ventricular assist device. In: Proceedings of 25 th Scientific Meeting, European Society for Magnetic Resonance in Medicine and Biology, Valencia, Spain, 2008:802.

25. Bock J, Kreher BW, Hennig J, Markl M. Optimized pre-processing of timeresolved 2D and 3D Phase Contrast MRI data. In: Proceedings of 15 Annual Meeting of ISMRM, Berlin, Germany 2007 (Program number 3138).

26. Kaczmarek I, Sachweh J, Groetzner J, Gulbins H, Mair H, Rainer KF, et al. Mechanical circulatory support in pediatric patients with the MEDOS assist device. ASAIO J. 2005;51:498-500.

27. Malaisrie SC, Pelletier MP, Yun JJ, Sharma K, Timek TA, Rosenthal DN, et al. Pneumatic paracorporeal ventricular assist device in infants and children: initial Stanford experience. J Heart Lung Transplant. 2008;27:173-7.

28. Letsou GV, Pate TD, Gohean JR, Kurusz M, Longoria RG, Kaiser L, et al. Improved left ventricular unloading and circulatory support with synchronized pulsatile left ventricular assistance compared with continuous-flow left ventricular assistance in an acute porcine left ventricular failure model. J Thorac Cardiovasc Surg. 2010;140:1181-8.

29. Pages O, Aubert S, Combes A, Luyt C, Pavie A, Léger P, et al. Paracorporeal pulsatile biventricular assist device versus extracorporal membrane oxygenation-extracorporal life support in adult fulminant myocarditis. J Thorac Cardiovasc Surg. 2009;137:194-19.

30. Crow S, John R, Boyle A, Shumway S, Liao K, Colvin-Adams M, et al. Gastrointestinal bleeding rates in recipients of nonpulsatile and pulsatile left ventricular assist devices. J Thorac Cardiovasc Surg. 2009;137:208-15. 